\title{
Plasmodium infection induces cross-reactive antibodies to carbohydrate epitopes on the SARS-CoV-2 Spike protein
}

Sarah Lapidus ${ }^{1,+}$, Feimei Liu ${ }^{2,+}$, Arnau Casanovas-Massana1 ${ }^{1}$ Yile Dai ${ }^{2}$, John D. Huck2, Carolina Lucas ${ }^{2}$, Jon Klein ${ }^{2}$, Renata B. Filler ${ }^{2,3}$, Madison S. Strine ${ }^{2,3}$, Mouhamad Sy ${ }^{4}$, Awa B. Deme ${ }^{4}$, Aida S. Badiane ${ }^{4}$, Baba Dieye ${ }^{4}$, Ibrahima Mbaye Ndiaye ${ }^{4}$, Younous Diedhiou $^{4}$, Amadou Moctar Mbaye ${ }^{4}$, Cheikh Tidiane Diagne ${ }^{5}$, Inés Vigan-Womas ${ }^{6}$, Alassane Mbengue ${ }^{7}$, Bacary D. Sadio ${ }^{8}$, Moussa M. Diagne ${ }^{8}$, Adam J. Moore ${ }^{1}$, Khadidiatou Mangou ${ }^{7}$, Fatoumata Diallo ${ }^{7}$, Seynabou D. Sene ${ }^{7}$, Mariama N. Pouye ${ }^{7}$, Rokhaya Faye $^{6}$, Babacar Diouf ${ }^{6}$, Nivison Nery $\mathrm{Jr}^{25,26}$, Federico Costa ${ }^{1,25,26}$, Mitermayer Reis ${ }^{1,25,26}$, M. Catherine Muenker ${ }^{1}$, Daniel Z. Hodson ${ }^{1}$, Yannick Mbarga ${ }^{22}$, Ben Z. Katz ${ }^{9}$, Jason R. Andrews ${ }^{10}$, Melissa Campbell ${ }^{11}$, Ariktha Srivathsan ${ }^{1}$, Kathy Kamath ${ }^{12}$, Elisabeth Baum-Jones ${ }^{12}$, Ousmane Faye ${ }^{8}$, Amadou Alpha Sall ${ }^{8}$, Juan Carlos Quintero Vélez $^{13,19,20}$, Michael Cappello ${ }^{14}$, Michael Wilson ${ }^{21}$, Choukri Ben-Mamoun ${ }^{15}$, Fabrice A. Somé ${ }^{16}$, Roch K. Dabiré ${ }^{16}$, Carole Else Eboumbou Moukoko ${ }^{23,24}$, Jean Bosco Ouédraogo ${ }^{16}$, Yap Boum II $^{17}$, John Shon ${ }^{12}$, Daouda Ndiaye ${ }^{4}$, Adam Wisnewski ${ }^{18}$, Sunil Parikh $^{1}$, Akiko Iwasaki ${ }^{2}$, Craig B. Wilen ${ }^{2}$, Albert I. Ko ${ }^{1,26}$, Aaron M. Ring ${ }^{2,+}$, and Amy K. Bei $^{1,4,7,+, *}$

\footnotetext{
${ }^{1}$ Yale School of Public Health, Department of Epidemiology of Microbial Diseases, New Haven, CT, 06510, USA

${ }^{2}$ Yale School of Medicine, Department of Immunobiology, New Haven, CT, 06510, USA

${ }^{3}$ Yale School of Medicine, Department of Laboratory Medicine, New Haven, CT, 06510, USA

${ }^{4}$ Cheikh Anta Diop University, Aristide le Dantec Hospital, Laboratory of Parasitology and Mycology, Dakar, Senegal

${ }^{5}$ DiaTROPIX Rapid Diagnostic Tests Facility, Institut Pasteur de Dakar, Dakar, Senegal

${ }^{6}$ Pôle Immunophysiopathologie et Maladies Infectieuses, Institut Pasteur de Dakar, Dakar, Senegal

${ }^{7}$ G4 - Malaria Experimental Genetic Approaches \& Vaccines, Pôle Immunophysiopathologie et Maladies

Infectieuses, Institut Pasteur de Dakar, Dakar, Senegal

${ }^{8}$ Pôle Virologie, Institut Pasteur de Dakar, Dakar, Senegal

${ }^{9}$ Department of Pediatrics, Northwestern University Feinberg School of Medicine, Division of Infectious Diseases, Ann \& Robert H. Lurie Children's Hospital of Chicago

${ }^{10}$ Division of Infectious Diseases and Geographic Medicine, School of Medicine, Stanford University, Stanford, CA, USA.

${ }^{11}$ Yale Center for Clinical Investigation, Yale School of Medicine, New Haven, Connecticut, USA

${ }^{12}$ Serimmune, Inc., Goleta, CA

${ }^{13}$ Grupo de Investigación Ciencias Veterinarias Centauro, Universidad de Antioquia, Medellin, Colombia

${ }^{14}$ Department of Pediatrics, Yale School of Medicine, New Haven, CT, USA

${ }^{15}$ Department of Internal Medicine, Section of Infectious Diseases, Yale School of Medicine, New Haven, CT, USA

${ }^{16}$ Institut de Recherche en Sciences de La Santé (IRSS)/Centre Muraz, Bobo-Dioulasso, Burkina Faso

${ }^{17}$ University of Yaoundé and Epicentre, Médecins Sans Frontières

${ }^{18}$ Yale Occupational and Environmental Medicine Program, Department of Internal Medicine, Yale School of

Medicine, New Haven, CT, USA

${ }^{19}$ Grupo de Investigación Ciencias Veterinarias Centauro, University of Antioquia, Medellín, Colombia

${ }^{20}$ Grupo de Investigación Microbiología Básica y Aplicada, University of Antioquia, Medellín, Colombia

${ }^{21}$ Department of Parasitology, Noguchi Memorial Institute for Medical Research, University of Ghana, Accra, Ghana

${ }^{22}$ Douala Military Hospital, Douala, Cameroon

${ }^{23}$ Department of Biological Sciences, Faculty of Medicine and Pharmaceutical Sciences, University of Douala, BP 2701 Douala, Cameroon

${ }^{24}$ Malaria Research Unit, Center Pasteur Cameroon, Yaoundé, Cameroon

${ }^{25}$ Instituto de Saúde Coletiva, Universidade Federal da Bahia, Salvador, BA, Brazil
} 
medRxiv preprint doi: https://doi.org/10.1101/2021.05.10.21256855; this version posted May 12, 2021. The copyright holder for this preprint

(which was not certified by peer review) is the author/funder, who has granted medRxiv a license to display the preprint in perpetuity.

It is made available under a CC-BY-NC-ND 4.0 International license .

\author{
${ }^{26}$ Instituto Gonçalo Moniz, Fundação Oswaldo Cruz, Ministério da Saúde, Salvador, BA, Brazil \\ *amy.bei@yale.edu \\ +these authors contributed equally to this work
}

\title{
ABSTRACT
}

Individuals with acute malaria infection generated high levels of antibodies that cross-react with the SARS-CoV-2 Spike protein. Cross-reactive antibodies specifically recognized the sialic acid moiety on N-linked glycans of the Spike protein and do not neutralize in vitro SARS-CoV-2. Sero-surveillance is critical for monitoring and projecting disease burden and risk during the pandemic; however, routine use of Spike protein-based assays may overestimate SARS-CoV-2 exposure and population-level immunity in malaria-endemic countries.

\section{Main}

Serological surveillance studies provide a fundamental understanding of past exposure to infectious diseases such as SARS$\mathrm{CoV}-2$. Recent serological surveillance studies reported out of Africa have often reported higher overall seroprevalence than is predicted based on case counts, prompting various hypotheses as to why seropositivity is higher than expected ${ }^{1-3}$.

We identified a high degree of cross-reactivity to SARS-CoV-2 Spike protein (S1 subunit) in individuals with acute (symptomatic and asymptomatic) malaria infection (Figure1A-1B) by ELISA. A total of 741 samples from 617 individuals from 8 countries collected before the first reported case of COVID-19 were tested for Spike protein seropositivity. The observed cross-reactivity was significantly higher in individuals with acute Plasmodium infection compared to uninfected individuals in malaria endemic areas (Figure1A-1B). Cross-reactivity was also significantly higher among uninfected individuals living in a malaria endemic setting with previous exposure compared to individuals in a non-endemic settings with no previous malaria exposure.

Though patterns of responses were generally similar for IgG and IgM, individuals with symptomatic malaria infection had significantly higher IgM but not IgG than asymptomatic individuals (Welch Two Sample t-test IgG p-value=0.077 and IgM p-value $<0.0001)$. These patterns remain after accounting for age group in a log-transformed multivariate linear regression model. Specifically, children with acute malaria infection had significantly higher normalized $\operatorname{IgG}$ and IgM than uninfected children in malaria endemic areas (both p-values $<0.0001$ ), and adults with acute infection had significantly higher normalized IgG and IgM than uninfected adults in endemic areas (IgG p-values=0.0047 and IgM p-value=0.0031). In S1-reactive antibody responses measured longitudinally in 131 samples from 21 subjects, IgG and IgM responses peaked between 0-4 weeks post infection for all patients, decreased with time, and were sometimes, though not consistently, boosted by subsequent reinfections (boosting in 1 of $4 \mathrm{IgG}$ samples and 2 of $4 \mathrm{IgM}$ samples with P. falciparum (Figure1C).

Of malaria positive subjects, 163 had Plasmodium falciparum infection (107 IgG positive and 98 IgM positive), 8 had $P$. malariae infection (6 IgG positive and $4 \mathrm{IgM}$ positive), 6 had $P$. falciparum/P. malariae mixed infections (3 IgG positive and 0 IgM positive), and 1 with $P$. vivax (0 IgG or IgM positive). Normalized IgG was significantly higher among subjects with $P$. falciparum, $P$. malariae, and mixed infections than among negative controls. Normalized IgM was only significantly higher among subjects with $P$. falciparum and mixed infections than among negative controls. However, the comparison was limited by few subjects with non- $P$. falciparum malaria. Normalized IgG and IgM was not significantly different between subjects with $P$. falciparum and subjects with $P$. malariae (Welch Two-Sample t-test p-value $=0.63$ for IgG and $\mathrm{p}$-value $=0.56$ for IgM). Thus, our results suggest that both $P$. falciparum and $P$. malariae induce higher $\operatorname{IgG}$ and possibly IgM reactivity.

Since both $P$. falciparum and SARS-CoV-2 infection can induce poly reactive B cells, ${ }^{4,5}$ we investigated this mechanism as a possible cause of SARS-CoV-2 reactivity. Sera from patients with Epstein Barr Virus (EBV), a disease with characteristic polyreactive $\mathrm{B}$ cells responses, were found to have significantly less reactivity than sera with acute malaria infections (t-test p-value $<0.0001$ for both $\operatorname{IgG}$ and IgM for EBV time-points averaging 6 weeks after infection and 6 months after infection), indicating that reactivity is not correlated with poly reactive B cells resulting from EBV infection (Figure1A).

In determining whether cross-reactivity was limited to S1 of SARS-CoV-2 or was observed with other SARS-CoV-2 proteins, we found limited correlated cross-reactivity between Spike S1 IgG and other SARS-CoV-2 proteins (baculovirus expressed Spike ectodomain: Pearson's $\mathrm{R}=0.062$, $\mathrm{p}$-value $=0.60$, and nucleocapsid: Pearson's $\mathrm{R}=0.17$, $\mathrm{p}$-value=0.15). We also did not find significant correlated cross-reactivity with a commercial test including S2 subunit and nucleocapsid combined (Omega Diagnostics) (Chi-squared with Yates correction p-value= 0.319).

To test whether the cross-reactivity in malaria endemic regions was related to exposure to other alpha and beta-human coronaviruses, we used bacterially expressed peptide arrays ${ }^{6-8}$ and Rapid Exoproteome Antigen Profiling (REAP) for Saccharomyces cerevisae expressed exoproteome array of receptor binding domains (RBD) from all seven human coronaviruses, namely 
SARS-CoV-2, NL63, HKU-1, 229E, SARS-CoV, MERS, and OC43. ${ }^{9}$ Although previous studies suggested cross-reactivity to SARS-CoV-2 is caused by prior exposure to NL63, $229 \mathrm{E}^{10}$ and OC43, ${ }^{11}$ we did not observe cross-reactivity between S1 positives by ELISA and peptides, SARS-CoV-2 RBD, or SARS-CoV-2 proteome using either approach except NL63, which showed overall high reactivity (using a REAP Score cutoff of 1.5, 35 of 48 individuals from Senegal and Burkina Faso tested positive [Figure2A]). No samples tested with REAP showed binding to SARS-CoV-2 Spike RBD. Linear peptide epitopes tested against bacterial display libraries did not identify significant binding of malaria-induced antibodies against SARS-CoV-2 peptides. Taken together, these data imply that the cross reactivity could be structural (in the N-terminal domain of S1 not including the RBD) or targeted to a post-translational modification such as carbohydrates.

To test the specificity of the cross-reactivity to either structural epitopes or glycosylation, we treated the S1 protein to alter its structure and composition of glycans and carbohydrates. In ELISAs, IgG cross-reactivity was maximally reversed upon treatment with neuraminidase, which removes the terminal sialic acids from glycosylated sites (decreased samples to 23.0\% [95\% CI: 1.1, 44.9]) S1 native OD, significantly more than SARS-CoV-2 convalescent serum (63\% of S1 native OD) (Figure2B). Although treatment with PNGase F (which removes all N-linked glycans) also significantly reduced reactivity (decreased samples to $31.3 \%$ [95\% CI: 14.3, 48.2] of OD of S1 native), the reduction was less than that of SARS-CoV-2 convalescent serum (decrease to $4.6 \%$ of OD of S1 native). Denaturing the S1 protein more modestly reduced reactivity (decreased samples to $71.6 \%$ [95\% CI: 59.1, 84.1] of OD of S1 native, SARS-CoV-2 convalescent serum decreased to 6.4\% of OD of S1 native). These results implicate cross-reacting antibody binding to terminal sialic acids of complex glycans. Of 22-N-linked glycosylation sites spanning the Spike protein, 52\% are complex and fucosylated and 15\% contain at least one sialic acid. ${ }^{12}$ The majority of the N-linked glycans in the S1 domain of Spike protein (aa16-685) are complex, and are both fucosylated and sialated. ${ }^{12}$ Three samples maintained high reactivity to S1 treated with neuraminidase $(133.6 \%, 126.2 \%$, and $131.2 \%$ of OD of S1 native), suggesting that cross-reactivity in these samples could be caused by another mechanism.

We next sought to determine whether these malaria-induced antibodies could neutralize SARS-CoV-2 invasion, possibly providing protection. We performed in vitro neutralization assays testing highly reactive samples using pseudotyped viruses (Vesicular stomatitis virus encoding a Renilla luciferase reporter gene pseudotyped with SARS-CoV-2 Spike) in dilutions from 1:10 to 1:1280 and SARS-CoV-2 viral isolates in Vero-E6 cell plaque assays in dilutions from 1:10 to 1:2430. Similar to a previous study that found no protection from preexisting cross-reactivity, ${ }^{11}$ samples with cross-reactive antibodies did not demonstrate significant neutralizing activity via invasion at any dilution in either experimental system (Figure2C).

Our study reveals that acute malaria infection can cause cross-reactivity to the S1 Spike protein through antibody binding to terminal sialic acids of complex glycans. Reactivity is less pronounced among people previously exposed to malaria but without acute infection. Cross-reactivity was not neutralizing, giving no evidence that malaria exposure protects against SARS-CoV-2 infection through antibody-mediated viral neutralization. Cross-reactivity has also been found in recent studies in malaria-endemic countries; two SARS-CoV-2 serological assays targeting the nucleocapsid protein had cross-reactivity among pre-pandemic samples from Nigeria with higher levels of malaria antibodies ${ }^{13}$. A high rate of false positives was also seen in pre-pandemic samples using commercially available ELISAs from Benin ${ }^{14}$ and from Ghana and Nigeria ${ }^{15}$.

These findings provide evidence of a target and mechanism for cross-reactivity and have implications for the global roll-out of serological surveillance tools that measure $\operatorname{IgG}$ or IgM reactive to the Spike protein. Serological surveys remain critical tools in understanding disease burden; however, cross-reactivity in malaria-endemic regions, and especially in patients with acute malaria, could lead to false positive antibody tests for individuals and overestimate population-level exposure. False positive results could cause an individual to underestimate their risk of future infection. At a population level, an overestimate of prior SARS-CoV-2 exposure could lead to overestimates of immunity, meaning larger proportions of people may remain susceptible to infection than serological surveillance might indicate, allowing for continued spread of SARS-CoV-2. Overestimates of exposure would also lead to underestimates of risk of severe disease among people exposed to SARS-CoV-2. Cross-reactive diagnostic tools may have limited utility for serological surveillance when predicting future re-emergence of COVID-19 in areas where incidence of COVID-19 is low relative to malaria. High rates of false positives could preclude using serological tests as correlates of immunity in malaria-endemic areas, hampering policy decision making with respect to risk stratification and implementation of non-pharmaceutical interventions. These results further highlight the need to validate diagnostics in populations with different disease exposures and optimize such diagnostics so that serological surveillance tools can accurately track SARS-CoV-2 exposure.

\section{References}

1. Chibwana, M. G. et al. High SARS-CoV-2 seroprevalence in health care workers but relatively low numbers of deaths in urban malawi. medRxiv DOI: 10.1101/2020.07.30.20164970 (2020).

2. Olayanju, O. et al. SARS-CoV-2 seropositivity in asymptomatic frontline health workers in ibadan, nigeria. Am J Trop Med Hyg DOI: 10.4269/ajtmh.20-1235 (2020). 
medRxiv preprint doi: https://doi.org/10.1101/2021.05.10.21256855; this version posted May 12, 2021. The copyright holder for this preprint

(which was not certified by peer review) is the author/funder, who has granted medRxiv a license to display the preprint in perpetuity.

It is made available under a CC-BY-NC-ND 4.0 International license .

3. Uyoga, S. et al. Seroprevalence of anti-SARS-CoV-2 IgG antibodies in kenyan blood donors. Science DOI: 10.1126/ science.abe1916 (2020).

4. Muellenbeck, M. F. et al. Atypical and classical memory b cells produce plasmodium falciparum neutralizing antibodies. $J$ Exp Med 210, 389-399, DOI: 10.1084/jem.20121970 (2013).

5. Wildner, N. H. et al. B cell analysis in SARS-CoV-2 versus malaria: Increased frequencies of plasmablasts and atypical memory b cells in COVID-19. J. Leukoc. Biol. 109, 77-90, DOI: https://doi.org/10.1002/JLB.5COVA0620-370RR (2021). _eprint: https://jlb.onlinelibrary.wiley.com/doi/pdf/10.1002/JLB.5COVA0620-370RR.

6. Pantazes, R. J. et al. Identification of disease-specific motifs in the antibody specificity repertoire via next-generation sequencing. Sci. Reports 6, 30312, DOI: 10.1038/srep30312 (2021). Number: 1 Publisher: Nature Publishing Group.

7. Haynes, W. A., Kamath, K., Daugherty, P. S. \& Shon, J. C. Protein-based immunome wide association studies (PIWAS) for the discovery of significant disease-associated antigens. bioRxiv 2020.03.18.997759, DOI: 10.1101/2020.03.18.997759 (2020). Publisher: Cold Spring Harbor Laboratory Section: New Results.

8. Kamath, K. et al. Antibody epitope repertoire analysis enables rapid antigen discovery and multiplex serology. Sci. Reports 10, 5294, DOI: 10.1038/s41598-020-62256-9 (2020). Number: 1 Publisher: Nature Publishing Group.

9. Wang, E. Y. et al. Diverse functional autoantibodies in patients with COVID-19. medRxiv DOI: 10.1101/2020.12.10. 20247205 (2020).

10. Tso, F. Y. et al. High prevalence of pre-existing serological cross-reactivity against severe acute respiratory syndrome coronavirus-2 (SARS-CoV-2) in sub-saharan africa. Int J Infect Dis 102, 577-583, DOI: 10.1016/j.ijid.2020.10.104 (2021).

11. Anderson, E. M. et al. Seasonal human coronavirus antibodies are boosted upon SARS-CoV-2 infection but not associated with protection. Cell 0, DOI: 10.1016/j.cell.2021.02.010 (2021). Publisher: Elsevier.

12. Watanabe, Y., Allen, J. D., Wrapp, D., McLellan, J. S. \& Crispin, M. Site-specific glycan analysis of the SARS-CoV-2 spike. Science 369, 330-333, DOI: 10.1126/science.abb9983 (2020). Publisher: American Association for the Advancement of Science Section: Report.

13. Steinhardt, L. C. et al. Cross-reactivity of two SARS-CoV-2 serological assays in a malaria-endemic setting. J. Clin. Microbiol. DOI: 10.1128/JCM.00514-21 (2021). Publisher: American Society for Microbiology Journals Section: Immunoassays.

14. Yadouleton, A. et al. Limited specificity of serologic tests for SARS-CoV-2 antibody detection, benin. Emerg Infect Dis 27, DOI: 10.3201/eid2701.203281 (2021).

15. Emmerich, P. et al. Limited specificity of commercially available SARS-CoV-2 IgG ELISAs in serum samples of african origin. Trop Med Int Heal. DOI: 10.1111/tmi.13569 (2021).

\section{Acknowledgements}

We would like to sincerely thank the study participants, clinical staff, and field teams for their dedicated involvement in this work. We acknowledge the contributions of Prof. Dyann F. Wirth at the Harvard TH Chan School of Public Health for her longstanding commitment to the collaboration between Harvard and UCAD and her continued mentorship. We are grateful to Elsio A. Wunder, Jr. for technical assistance and coordination for the project.

Funding: This work was supported by the Fogarty International Center of the NIH (K01 TW010496), Yale School of Public Health Start-up funds, and G4 group funding (G45267, Malaria Experimental Genetic Approaches \& Vaccines) from the Institut Pasteur de Paris and Agence Universitaire de la Francophonie (AUF) to AKB, NIH grants K08 AI128043 (CBW), R01 AI148467 (CBW), DGE1752134 (MSS); a Burroughs Wellcome Fund Career Award for Medical Scientists (CBW) the Ludwig Family Foundation (CBW), the Mathers Charitable Foundation (CBW); an Emergent Ventures fast grant (CBW).

\section{Data Accessibility}

Data associated with this manuscript can be found at: https://doi.org/10.5061/dryad.w6m905qpj

\section{Author contributions statement}

$\mathrm{AKB}, \mathrm{AIK}$, and AMR conceived and directed the project, SL and FL performed the experiments, SL analyzed the data, ACM, YD, JDH, CL, JK, RBF, MSS, MS, ABD, ASB, BD, IMN, YD, AMM, CTD, IVW, AM, BDS, MMD, AJM, KM, FD, SDS, MNP, RF, BD, NN, FC, MR, MCM, DZH, YM, BZK, JRA, MC, AS, KK, EBJ, OF, AAS, JCQV, MC, MW, CBM, FAS, RKD, CEEM, JBO, YB2, JS, DN, AW, SP, AI, CBW, AIK, AMR, AKB performed data acquisition, data analysis and interpretation. 
medRxiv preprint doi: https://doi.org/10.1101/2021.05.10.21256855; this version posted May 12, 2021. The copyright holder for this preprint

(which was not certified by peer review) is the author/funder, who has granted medRxiv a license to display the preprint in perpetuity.

It is made available under a CC-BY-NC-ND 4.0 International license .

AKB and SL wrote the manuscript. All authors have read, provided feedback, and approved the submitted version of the manuscript.

\section{Additional information}

Competing interests. A.M.R. and Y.D. (Yale University) are named inventors on a patent application describing the REAP technology. A.M.R. is the founder of Seranova Bio. C.B.W. (Yale University)has a patent pending entitled "Compounds and Compositions for Treating, Ameliorating, and/or Preventing SARS-CoV-2 Infection and/or Complications Thereof". KK, EBJ, and JS receive salary and hold stock options from Serimmune Inc.

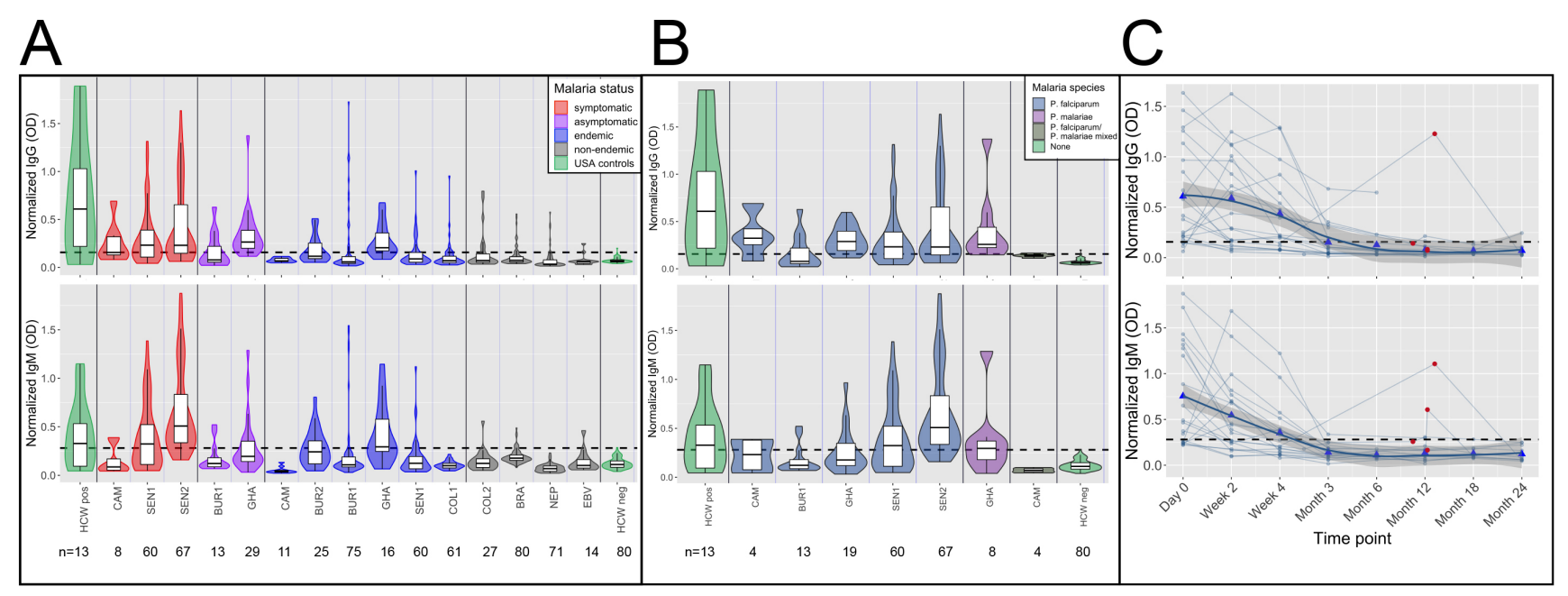

Figure 1. High frequency of cross-reactive antibodies to SARS-CoV-2 Spike protein from Plasmodium-infected individuals. In A. and B., Violin plots showing normalized IgG and IgM responses among patients from different cohorts. A. Among all subjects, those in non-malaria endemic areas had significantly lower IgG and IgM than others (t-test IgG p-value $<0.0001$ and IgM p-value $<0.0001$.) Subjects with acute malaria infection (symptomatic and asymptomatic) had significantly higher IgG and IgM than uninfected subjects living in malaria endemic areas (t-test p-value $<0.0001$ for both IgG and $\operatorname{IgM}$ ). B. Normalized IgG was significantly higher among subjects with P. falciparum, P. malariae, and mixed infections than among negative controls (Welch Two Sample t-test $\mathrm{p}$-value $<0.0001$ for $P$. falciparum, $\mathrm{p}$-value $=0.044$ for $P$. malariae and $\mathrm{p}$-value $=0.008$ for mixed infections), and normalized $\operatorname{IgM}$ was significantly higher among subjects with $P$. falciparum and mixed infections but not $P$. malariae than among negative controls (Welch Two Sample t-test $\mathrm{p}$-value $<0.0001$ for $P$. falciparum, $\mathrm{p}$-value $=0.106$ for $P$. malariae, and $\mathrm{p}$-value $=0.018$ for mixed infections). C. Normalized $\operatorname{IgG}$ and $\operatorname{IgM}$ over time in 21 subjects with $P$. falciparum monoinfection on Day 0. Both $\operatorname{IgG}$ and $\operatorname{IgM}$ peaked between Day 0 and Week 4 for all subjects. Reinfection, shown by red circles, boosted IgG response in 1 of 4 subjects and IgM response in 2 of 4 subjects. Bold trend line based on local regression (LOESS). In A. B. and C., normalized IgG or IgM calculated by IgG or IgM OD divided by IgG or IgM of positive control (camelid monoclonal chimeric nanobody VHH72 antibody was IgG control, and pooled convalescent serum from SARS-CoV-2 patients was IgM control). Black dashed lines represent cutoffs for positivity, calculated from normalized IgG and IgM values from 80 RT-qPCR negative HCWs (mean + 3 SDs). 
medRxiv preprint doi: https://doi.org/10.1101/2021.05.10.21256855; this version posted May 12, 2021. The copyright holder for this preprint (which was not certified by peer review) is the author/funder, who has granted medRxiv a license to display the preprint in perpetuity.

It is made available under a CC-BY-NC-ND 4.0 International license .

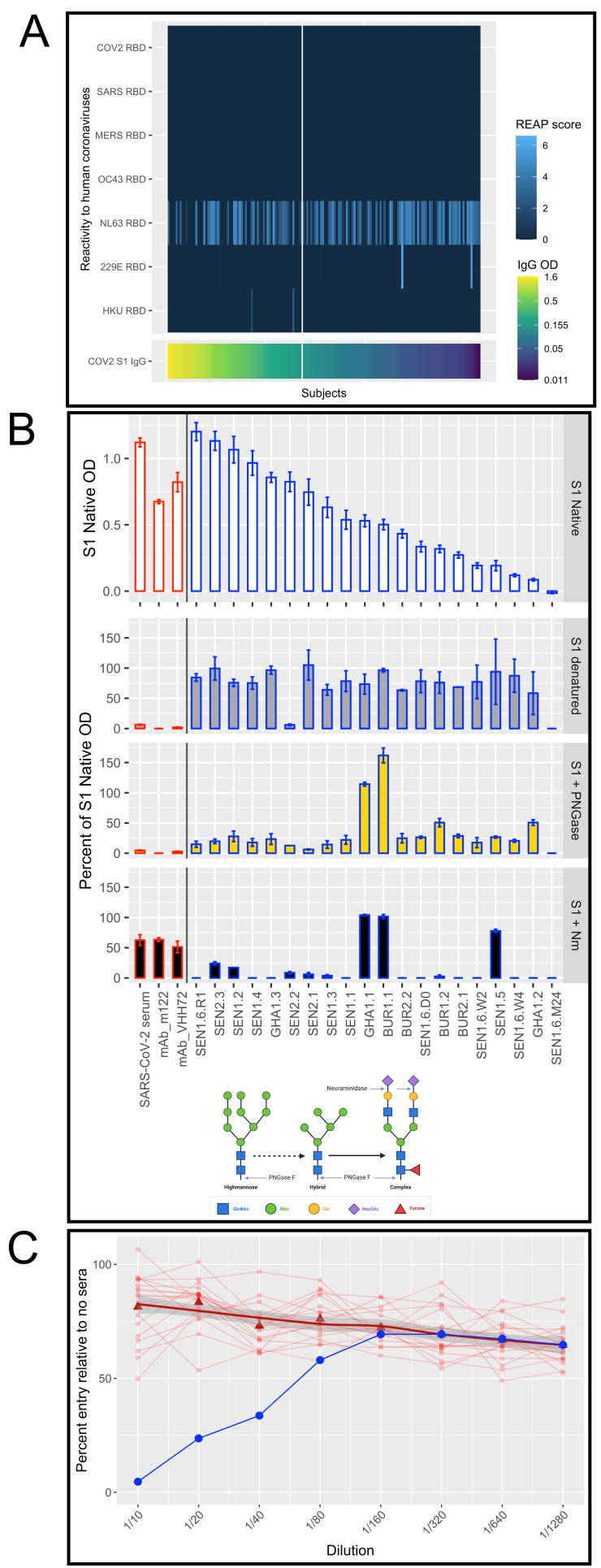

Figure 2. Cross-reactive IgG targets sialic acid on $\mathbf{N}$-linked glycans and is not due to cross-reactivity with other coronaviruses. A. Cross-reactivity to human coronaviruses is only observed for NL63 and differed between S1 positive and S1 negative subjects (S1 positives on the left side of the vertical white line and S1 negatives on the right side) in REAP testing using a REAP score cutoff of 1.5. B. S1 protein was subjected to three conditions to modify the structure: denaturing; treating with PNGase F to remove all N-linked glycans; and treating with Neuraminidase to remove sialic acid. ELISA was performed on each protein condition and the figure shows percent of S1 Native OD under each condition for 3 controls (pooled convalescent serum from SARS-CoV-2 patients, mouse monoclonal M122 antibody, and camelid monoclonal chimeric nanobody VHH72 antibody, all outlined in red) and 20 subjects (outlined in black). Neuraminidase treatment decreased samples to $23.0 \%$ (95\% CI: 1.1, 44.9) of OD of S1 native, significantly more than SARS-CoV-2 convalescent serum (decreased to $63 \%$ of OD of S1 native), suggesting cross-reactivity was due to reactions with terminal sialic from glycosylated sites. C. In a neutralizing assay using pseudotyped viruses (VSV-Renilla luciferase pseudotyped with Spike), 20 samples (red circles) w6it: high S1 Spike IgG ELISA reactivity showed no neutralization. Bold trend line based on local regression (LOESS) of samples. Control (serum from a COVID-19 positive inpatient, in blue) shows neutralization at dilutions of 1/40 and less. 